\title{
SURJECTIVITY OF DIFFERENTIAL OPERATORS AND LINEAR TOPOLOGICAL INVARIANTS FOR SPACES OF ZERO SOLUTIONS
}

\author{
T. KALMES
}

\begin{abstract}
We provide a sufficient condition for a linear differential operator with constant coefficients $P(D)$ to be surjective on $C^{\infty}(X)$ and $\mathscr{D}^{\prime}(X)$, respectively, where $X \subseteq \mathbb{R}^{d}$ is open. Moreover, for certain differential operators this sufficient condition is also necessary and thus a characterization of surjectivity for such differential operators on $C^{\infty}(X)$, resp. on $\mathscr{D}^{\prime}(X)$, is derived. Additionally, we obtain for certain surjective differential operators $P(D)$ on $C^{\infty}(X)$, resp. $\mathscr{D}^{\prime}(X)$, that the spaces of zero solutions $C_{P}^{\infty}(X)=$ $\left\{u \in C^{\infty}(X) ; P(D) u=0\right\}$, resp. $\mathscr{D}_{P}^{\prime}(X)=\left\{u \in \mathscr{D}^{\prime}(X) ; P(D) u=0\right\}$ possess the linear topological invariant $(\Omega)$ introduced by Vogt and Wagner in [27], resp. its generalization $(P \Omega)$ introduced by Bonet and Domański in [1].
\end{abstract}

\begin{abstract}
Keywords: Surjectivity of differential operator; Linear topological invariants for kernels of differential operators; Differential operators on vector-valued spaces of functions and distributions; Parameter dependence for solutions of linear partial differential equations
\end{abstract}

2010 MSC: Primary: 35E10, 46A63. Secondary: 35E20

\section{IntRODUCTION}

A classical result by Malgrange [14, Chapitre 1, Théorème 4] from 1955 states that for a polynomial $P \in \mathbb{C}\left[X_{1}, \ldots, X_{n}\right]$ and for an open set $X \subseteq \mathbb{R}^{n}$ the constant coefficient differential operator $P(D): C^{\infty}(X) \rightarrow C^{\infty}(X)$ is surjective if and only if $X$ is $P$-convex for supports, that is, if and only if for every compact subset $K$ of $X$ there is another compact subset $L$ of $X$ such that for each compactly supported distribution $u \in \mathscr{E}^{\prime}(X)$ with $\operatorname{supp} P(-D) u \subseteq K$ it holds supp $u \subseteq L$.

Although this characterization is more than 60 years old, there are very few differential operators $P(D)$ for which there is a satisfactory geometric evaluation of this condition for open $X \subseteq \mathbb{R}^{n}$. Convex open sets are $P$-convex for supports, whenever $P \neq 0$, every open set is $P$-convex for supports whenever $P$ is elliptic, and there is a complete geometric characterization of $P$-convexity for supports in the two dimensional case due to Hörmander [10, Theorem 10.8.3]. In arbitrary dimensions however, the problem of characterising open subsets of $\mathbb{R}^{n}$ which are $P$-convex for supports is far from being solved. For second order operators with principal part equal to the wave operator there is a characterization [10, Theorem 10.8.6] which is essentially due to Persson [19] who extended this result in [20] to arbitrary operators of real principle type but only in $\mathbb{R}^{3}$, while Tintarev [22], [21] evaluated $P$-convexity for supports for operators of real principal type for bounded open sets $X$ with analytic boundary, and for open sets $X$ whose boundary does not contain any straight line, respectively. Moreover, Nakane [18] gave a geometric characterization when $X$ is $P$-convex for supports for the special case of polynomials $P$ acting along a subspace and being elliptic there. A more convenient geometric

E-mail address: thomas.kalmes@mathematik.tu-chemnitz.de. 
characterization for this class of operators can be found in [10, Theorem 10.8.5]. Apart from these very special classes of polynomials, resp. operators, not much is known.

In 1962, Hörmander showed [8] that $P(D)$ is surjective on $\mathscr{D}^{\prime}(X)$ if and only if $X$ is $P$-convex for supports as well as $P$-convex for singular supports, where the latter means that for every compact subset $K$ of $X$ there is another compact subset $L$ of $X$ such that for each $u \in \mathscr{E}^{\prime}(X)$ with sing supp $P(-D) u \subseteq K$ it follows that sing supp $u \subseteq L$. Thus, surjectivity of $P(D)$ on $\mathscr{D}^{\prime}(X)$ implies surjectivity of $P(D)$ on $C^{\infty}(X)$, but in general the converse implication is far from being true, except in case of $X \subseteq \mathbb{R}^{2}$ as was recently shown in [11]. There, the author gives a positive solution of a conjecture by Trèves [23, p. 389, Problem 2] showing that for open $X \subseteq \mathbb{R}^{2} P$-convexity for supports implies $P$-convexity for singular supports. Though better understood than $P$-convexity for supports, giving geometric characterizations for $P$-convexity for singular supports for concrete differential operators $P(D)$ is not a triviality, see e.g. [10, Chapter 10], [9], [5], [11], and [12].

Despite the lack of a satisfactory characterization of $P$-convexity for (singular) supports in many cases of operators and thus a characterization of general solvability of linear partial differential equations $P(D) u=f$ for arbitary smooth functions/distributions $f$ on open subsets $X \subseteq \mathbb{R}^{n}$ there is a number of results, both classical and very recent, by several authors considering the question whether surjectivity of $P(D)$ on $C^{\infty}(X)$, resp. $\mathscr{D}^{\prime}(X)$ passes on to surjectivity of $P(D)$ on the space of vector-valued smooth functions $C^{\infty}(X, E)$, resp. vector-valued distributions $\mathscr{D}^{\prime}(X, E)$, where $E$ is a locally convex space (see, e.g. [7], [28], [1], [4], [2]). Clearly, this question has a positive answer whenever $P(D)$ admits a continuous linear right inverse which was characterized by Meise, Taylor, and Vogt in [15] (see also [16]) solving a problem posed by Schwartz.

In case $E$ is a space of functions or distributions itself, surjectivity of $P(D)$ on $C^{\infty}(X, E)$, resp. $\mathscr{D}^{\prime}(X, E)$ is equivalent to an affirmative answer to the problem of parameter dependence for solutions of the differential equation $P(D) u_{\lambda}=f_{\lambda}$, i.e. to the problem whether for a given family $f_{\lambda}$ of smooth functions, resp. distributions on $X$ depending on a parameter $\lambda$ such that the function/distribution $\lambda \mapsto f_{\lambda}(x)$ belongs to $E$ it is always possible to find solutions $u_{\lambda}$ such that $\lambda \mapsto u_{\lambda}(x)$ also belongs to $E$.

For Fréchet spaces $E$ it follows from a result of Grothendieck [7] that for a surjective differential operator $P(D)$ on $C^{\infty}(X)$ the operator is also surjective on $C^{\infty}(X, E)$. The same implication is no longer true in general when $E$ is the strong dual of a Fréchet space $F$, as has been shown by Vogt [28]. However, Vogt also showed that in case of $F=s$, with $s$ denoting the space of rapidly decreasing sequences, surjectivity of $P(D)$ on $C^{\infty}(X)$ passes on to surjectivity on $C^{\infty}\left(X, s^{\prime}\right)$ if and only if the kernel of $P(D)$ in $C^{\infty}(X)$, which we denote by $C_{P}^{\infty}(X)$ and which is equipped with the Fréchet space structure inherited by $C^{\infty}(X)$, has the linear topological invariant $(\Omega)$ (see [28, Proposition 2.2, Theorem 2.4]). Recall that a Fréchet space $F$ with a fundamental system of seminorms $\|\cdot\|_{1} \leq\|\cdot\|_{2} \leq \ldots$ is said to have $(\Omega)$ if the following holds

$$
\forall k \exists l \forall m \exists \theta \in(0,1), C>0 \forall u \in F^{\prime}:\|u\|_{l}^{*} \leq C\|u\|_{k}^{*(1-\theta)}\|u\|_{m}^{* \theta},
$$

where $\|\cdot\|_{k}^{*}$ etc. denote the so-called dual norm on $F^{\prime}$ to $\|\cdot\|_{k}$, i.e. $\|u\|_{k}^{*}=$ $\sup \left\{|u(x)| ; x \in E,\|x\|_{k} \leq 1\right\}$. Obviously, property $(\Omega)$ is a linear topological invariant, in particular it is independent of the special choice of fundamental system of seminorms on $E$. 
For hypoelliptic polynomials $P$ property $(\Omega)$ can be viewed as an abstract qualitative version of Hadamard's three circles theorem. In fact, having in mind Grothendieck-Köthe duality (see e.g. [6], [28, p. 175], [31]) by which for surjective $P(D)$ on $C^{\infty}(X)$ the strong dual space of $C_{P}^{\infty}(X)$ is given in a natural way by the space of germs of smooth functions $u$ on the complement of $X$ which satisfy $P(-D) u=0$ and which behave well at infinity and taking into account that in the case of hypoelliptic $P(D)$ the dual norms appearing in (1) can be chosen as (polynomially) weighted supremum norms over the complement of compact subsets of $X$, condition (1) becomes in fact a very concrete generalisation of Hadamard's result for the Cauchy-Riemann operator to arbitrary hypoelliptic differential operators.

Apart from being characteristic for when surjectivity of $P(D)$ on $C^{\infty}(X)$ passes on to surjectivity of $P(D)$ on $C^{\infty}\left(X, s^{\prime}\right)$ it has also been shown by Vogt in [28] that property $(\Omega)$ of $C_{P}^{\infty}(X)$ is sufficient for $P(D)$ to be surjective on $C^{\infty}\left(X, F^{\prime}\right)$ provided that the Fréchet space $F$ has property $(D N)$, i.e. the following condition holds

$$
\exists k \forall l \exists m, C>0 \forall x \in F:\|x\|_{l}^{2} \leq C\|x\|_{k}\|x\|_{m},
$$

where $\|\cdot\|_{1} \leq\|\cdot\|_{2} \leq \ldots$ is again a fundamental system of seminorms. Examples of Fréchet spaces with $(D N)$ are $s, \mathscr{S}\left(\mathbb{R}^{n}\right)$ the space rapidly decreasing functions, $C_{2 \pi}^{\infty}\left(\mathbb{R}^{m}\right)$ the space of smooth functions which are $2 \pi$-periodic in each variable, $H(\mathbb{C})$ the space of entire functions, or more generally Fréchet power series spaces of infinite type $\Lambda_{\infty}(\alpha)$ (see e.g. [17]).

In [28] it has been proved that for elliptic operators $P$ the kernel $C_{P}^{\infty}(X)$ always has $(\Omega)$ and that the same holds in the more general case of hypoelliptic $P$ but under the restriction that the open set $X \subseteq \mathbb{R}^{n}$ is convex. Therefore it was conjectured for a long time that for a hypoelliptic operator $P(D)$ which is surjective on $C^{\infty}(X)$ its kernel $C_{P}^{\infty}(X)$ always has $(\Omega)$ independent of $X$ being convex or not. However, only recently, this conjecture has been settled in the negative. In [13] for any $n \geq 3$ an example of a hypoelliptic operator $P(D)$ and a $P$-convex open set $X \subseteq \mathbb{R}^{n}$ has been given such that $C_{P}^{\infty}(X)$ does not have $(\Omega)$. (That $n \geq 3$ is essential here follows from results obtained in [12].) Stronger variants of the linear topological invariant $(\Omega)$ for $C_{P}^{\infty}(X)$ have been considered in [30].

It will be shown in section 4 below that the kernels $C_{P}^{\infty}(X)$ of semi-elliptic differential operators $P(D)$ for which the zero set of the principal part is a one dimensional subspace of $\mathbb{R}^{n}$ always has $(\Omega)$ whenever $X$ is $P$-convex. This class of differential operators contains in particular non-degenerate parabolic operators which is obviously of particular interest in view of applications to concrete problems.

The problem of surjectivity of $P(D)$ on the space of vector-valued distributions $\mathscr{D}^{\prime}(X, E)$ instead of vector-valued smooth functions was only addressed recently by Bonet and Domański in [1]. They proved that for a surjective differential operator $P(D)$ on $\mathscr{D}^{\prime}(X)$ the operator $P(D)$ is surjective on $\mathscr{D}^{\prime}\left(X, F^{\prime}\right)$ for a nuclear Fréchet space $F$ with property $(D N)$ if the kernel of $P(D)$ in $\mathscr{D}^{\prime}(X)$, which we denote by $\mathscr{D}_{P}^{\prime}(X)$ and which we equip with the locally convex topology inherited by the strong topology on $\mathscr{D}^{\prime}(X)$, has property $(P \Omega)$, a generalisation of $(\Omega)$ to the category of PLS-spaces. Recall that a locally convex space $E$ is a PLS-space if $E$ is the projective limit of a sequence of strong duals of Fréchet-Schwartz spaces, $E=$ $\operatorname{proj}_{N \in \mathbb{N}} X_{N}, X_{N}=\operatorname{ind}_{n \in \mathbb{N}} X_{N, n}$, where $X_{N, n}$ are Banach spaces and the linking maps in the inductive limit $\operatorname{ind}_{n \in \mathbb{N}} X_{N, n}$ are compact. A PLS-space $E$ has property $(P \Omega)$ if

$$
\begin{aligned}
& \forall K \exists L \geq K \forall M \geq L \exists k \forall l \exists m, C>0, \theta \in(0,1) \forall u \in X_{K}^{\prime}: \\
& \left\|u^{\prime} \circ i_{K}^{L}\right\|_{L, l}^{*} \leq C\left\|u^{\prime} \circ i_{K}^{M}\right\|_{M, m}^{*(1-\theta)} \max \left\{\left\|u^{\prime}\right\|_{K, k}^{* \theta},\left\|u^{\prime} \circ i_{K}^{M}\right\|_{M, m}^{* \theta}\right\},
\end{aligned}
$$


where $\|\cdot\|_{L, l}^{*}$ denotes the dual norm to the norm on the Banach space $X_{L, l}$ etc. and $i_{K}^{L}$ denotes the inclusion from $X_{K}$ into $X_{L}$. It is well-known that Fréchet-Schwartz spaces are PLS-spaces and examples of PLS-spaces are thus spaces of holomorphic functions $H(V)$, smooth functions $C^{\infty}(X)$, or more general spaces of distributions $\mathscr{D}^{\prime}(X)$, real analytic functions $\mathscr{A}(X)$, ultradistributions in the sense of Beurling $\mathscr{D}_{(\omega)}^{\prime}(X)$, or ultradifferentiable functions in the sense of Roumieu $\mathscr{E}_{\{\omega\}}(X)$. See e.g. [3] for more on PLS-spaces, their properties, and their importance in analysis. It is shown in [1] that a Fréchet-Schwartz space has $(P \Omega)$ if and only if it has $(\Omega)$. Recall that for hypoelliptic differential operators $P(D)$ the kernels $C_{P}^{\infty}(X)$ and $\mathscr{D}_{P}^{\prime}(X)$ coincide as locally convex spaces by $[25$, Theorem 52.1$]$ so that $\mathscr{D}_{P}^{\prime}(X)$ is a Fréchet-Schwartz space and thus it has $(P \Omega)$ if and only if it has $(\Omega)$.

The present article is organized as follows. In section 2 we give sufficient conditions for $P$-convexity for (singular) supports for $X$ in terms of the minimum principle for the boundary distance of $X$ being valid in certain affine subspaces related to $P$. This enables to give geometric characterizations for $X$ for the surjectivity of $P(D)$ on $C^{\infty}(X)$ and $\mathscr{D}^{\prime}(X)$, respectively, for certain types of differential operators.

In section 3 the sufficient condition for $P$-convexity for singular supports is used to give for certain differential operators a positive solution to the problem whether for a surjective operator $P(D)$ on $\mathscr{D}^{\prime}(X)$ the augmented operator $P^{+}(D)$ is surjective on $\mathscr{D}^{\prime}(X \times \mathbb{R})$, where $P^{+}\left(x_{1}, \ldots, x_{n+1}\right)=P\left(x_{1}, \ldots, x_{n}\right)$. This problem was posed by Bonet and Domański in [1]. Although in general this problem has a negative solution as shown in [13], in section 3 we show that this problem has always a positive solution for certain semi-elliptic differential operators including the heat operator, and for operators acting along a subspace of $\mathbb{R}^{n}$ and being elliptic there.

In section 4 we apply the results from section 3 to the differential operators considered there in order to show that the spaces of zero solutions $C_{P}^{\infty}(X)$, resp. $\mathscr{D}_{P}^{\prime}(X)$ have property $(\Omega)$, resp. $(P \Omega)$ whenever $P(D)$ is surjective on $C^{\infty}(X)$, resp. $\mathscr{D}^{\prime}(X)$ so that for certain locally convex spaces $E P(D)$ is also surjective on $C^{\infty}(X, E)$, resp. on $\mathscr{D}^{\prime}(X, E)$.

Throughout the paper we use standard notation from the theory of partial differential operators, see e.g. [10], and functional analysis, see e.g. [17].

\section{Conditions for $P$-COnvexity}

It is well-known that a necessary condition for an open subset $X \subseteq \mathbb{R}^{n}$ to be $P$-convex for supports, respectively $P$-convex for singular supports, is that the boundary distance of $X$ satisfies the minimum principle in every characteristic hyperplane, respectively in certain affine subspaces related to $P$, resp. (cf. [10, Theorem 10.8.1, Corollary 11.3.2, resp.]). Recall that a real-valued function $f$ on $X \subseteq \mathbb{R}^{n}$ is said to satisfy the minimum principle in a closed subset $F$ of $\mathbb{R}^{n}$ if for every compact set $K \subseteq F \cap X$ we have

$$
\min _{x \in K} f(x)=\min _{\partial_{F} K} f(x),
$$

where $\partial_{F} K$ denotes the boundary of $K$ in $F$. We set the boundary distance $d_{X}$ of $X$ to be the mapping

$$
d_{X}: X \rightarrow \mathbb{R}, x \mapsto \operatorname{dist}\left(x, X^{c}\right),
$$

where the distance is taken with respect to the Euclidean norm $|x|$ in $\mathbb{R}^{n}$. The next theorem gives sufficient conditions for $P$-convexity for (singular) supports in terms of $d_{X}$ satisfying the minimum principle in certain affine subspaces related to $P$, and it is the main result of this section. In its formulation we use the following functional defined on the subspaces of $\mathbb{R}^{n}$. It was introduced by Hörmander in 
the context of continuation of differentiability for solutions of partial differential equations, cf. [10, Section 11.3].

For a subspace $V \subseteq \mathbb{R}^{n}$ we set

$$
\sigma_{P}(V)=\inf _{t \geq 1} \liminf _{\xi \rightarrow \infty} \frac{\tilde{P}_{V}(\xi, t)}{\tilde{P}(\xi, t)}
$$

where for $t \geq 1$ and $\xi \in \mathbb{R}^{n}$

$$
\tilde{P}_{V}(\xi, t):=\sup _{x \in V,|x| \leq t}|P(x+\xi)|, \tilde{P}(\xi, t):=\tilde{P}_{\mathbb{R}^{n}}(\xi, t) .
$$

Moreover, for $x \in \mathbb{R}^{n}$ we use the abbreviation $\sigma_{P}(x):=\sigma_{P}(\operatorname{span}\{x\})$.

Theorem 1. Let $P$ be a polynomial with principal part $P_{m}$ and let $X \subseteq \mathbb{R}^{n}$ be open. Moreover, let $W \neq\{0\}$ be a subspace of $\mathbb{R}^{n}$ such that $d_{X}$ satisfies the minimum principle in $x+W$ for every $x \in \mathbb{R}^{n}$.

i) If $\left\{x \in \mathbb{R}^{n} ; P_{m}(x)=0\right\} \subseteq W^{\perp}$ then $X$ is $P$-convex for supports.

ii) If $\left\{x \in \mathbb{R}^{n} ; \sigma_{P}(x)=0\right\} \subseteq W^{\perp}$ then $X$ is $P$-convex for singular supports.

With $W=\mathbb{R}^{n}$ part i) of the above theorem gives a new proof of the well-known result that for elliptic $P$ every open set $X \subseteq \mathbb{R}^{n}$ is $P$-convex for supports. $P$ convexity for supports for polynomials for which the zero set of its principal part is contained in a non-trivial subspace of $\mathbb{R}^{n}$ was also considered by Zachmanoglou [32]. In order to prove Theorem 1 we first give a useful characterization of when $d_{X}$ satisfies the minimum principal in every affine subspace parallel to a given subspace $W$. Therefore, we introduce the following notion.

Definition 2. Let $W$ be a subspace of $\mathbb{R}^{n}$ and $X \subseteq \mathbb{R}^{n}$. For an interval $I \subseteq \mathbb{R}$ a curve $\gamma: I \rightarrow X$ is called $W$-regular if $\gamma$ is continuous and piecewise continuously differentiable with $\gamma^{\prime}(t) \in W$ for all $t \in I$ where $\gamma$ is differentiable.

For $X \subseteq \mathbb{R}^{n}$ we denote by $\partial_{\infty} X$ the boundary of $X$ in the one-point compactification of $\mathbb{R}^{n}$, thus $\infty \in \partial_{\infty} X$ whenever $X$ is an unbounded subset of $\mathbb{R}^{n}$. For a curve $\gamma: I \rightarrow X$ we set $|\gamma|:=\gamma(I)$

Proposition 3. Let $W$ be a subspace of $\mathbb{R}^{n}, X \subseteq \mathbb{R}^{n}$ be open, $K \subseteq X$ be compact and non-empty, and $x \in X \backslash K$ such that $|\gamma| \cap K \neq \emptyset$ for every $W$-regular curve $\gamma:[0, \infty) \rightarrow X$ with $\gamma(0)=x$ and $\liminf _{t \rightarrow \infty} \operatorname{dist}\left(\gamma(t), \partial_{\infty} X\right)=0$.

Then, the connected component $Z$ in $(x+W) \cap(X \backslash K)$ which contains $x$ is bounded and $\partial_{x+W} Z$, the boundary of $Z$ in $x+W$, is contained in $K \cap(x+W)$.

Proof. Assume that $Z$ is unbounded. For notational convenience let us define $V:=$ $(x+W) \cap(X \backslash K)$. Because $V$ is locally pathwise connected and $Z$ is open in $V$, thus locally pathwise connected, too, the connectedness of $Z$ implies that $Z$ is pathwise connected. Hence, there is a continuous, piecewise continuously differentiable curve $\gamma:[0, \infty) \rightarrow Z$ with $\gamma(0)=x$ and $\lim _{t \rightarrow \infty}|\gamma(t)|=\infty$. Because $Z \subseteq X \backslash K$ this contradicts the hypothesis.

Thus, $Z$ is a bounded subset of $V$. Because the inclusion $V \hookrightarrow x+W$ is a homeomorphism onto its image and because $Z$ is open and closed in $V$ so that 
$\partial_{V} Z=\emptyset$, we have

$$
\begin{aligned}
\partial_{x+W} Z= & \left(\left(\partial_{x+W} Z\right) \cap V\right) \cup\left(\left(\partial_{x+W} Z\right) \cap(K \cap(x+W))\right) \\
& \cup\left(\left(\partial_{x+W} Z\right) \cap\left(\left(\mathbb{R}^{n} \backslash X\right) \cap(x+W)\right)\right) \\
= & \partial_{V} Z \cup\left(\left(\partial_{x+W} Z\right) \cap(K \cap(x+W))\right) \\
& \cup\left(\left(\partial_{x+W} Z\right) \cap\left(\left(\mathbb{R}^{n} \backslash X\right) \cap(x+W)\right)\right) \\
= & \left(\left(\partial_{x+W} Z\right) \cap(K \cap(x+W))\right) \\
& \cup\left(\left(\partial_{x+W} Z\right) \cap\left(\left(\mathbb{R}^{n} \backslash X\right) \cap(x+W)\right)\right) .
\end{aligned}
$$

So, if we can show that $\left(\partial_{x+W} Z\right) \cap\left(\left(\mathbb{R}^{n} \backslash X\right) \cap(x+W)\right)=\emptyset$ the proposition is proved.

Assume that there is

$$
y \in\left(\partial_{x+W} Z\right) \cap\left(\left(\mathbb{R}^{n} \backslash X\right) \cap(x+W)\right) .
$$

Because $V \hookrightarrow x+W$ is a homeomorphism onto its image it follows that $Z$ is a pathwise connected subset of $x+W$. Denote by $C$ the closure of $Z$ in $x+W$. Since $Z$ is open in $x+W$ it follows that there is a continuous, piecewise continuously differentiable curve $\gamma:[0, \infty) \rightarrow Z$ with $\gamma(0)=x$ and $\lim _{t \rightarrow \infty} \gamma(t)=y$.

Indeed, there is $y_{1}$ in $B(y, 1) \cap Z$ and a continuous, piecewise continuously differentiable $\alpha_{1}:[0,1] \rightarrow Z$ with $\alpha_{1}(0)=x, \alpha_{1}(1)=y_{1}$. Next, as we can find $y_{2} \in B\left(y, 1-\left|y_{1}\right|\right) \cap Z$ there is a continuous, piecewise continuously differentiable $\alpha_{2}:[1,2] \rightarrow Z$ with $\alpha_{2}(1)=y_{1}$ and $\alpha_{2}(2)=y_{j}$. Preceding in this way we obtain a sequence $\left(y_{j}\right)_{j \in \mathbb{N}}$ in $Z$ with $y=\lim _{j \rightarrow \infty} y_{j}$ and a sequence of continuous, piecewise continuously differentiable curves $\alpha_{j}:[j-1, j] \rightarrow Z$ with $\alpha_{j}(j-1)=y_{j-1}$ and $\alpha_{j}(j)=y_{j}$. Joining these curves gives the desired $\gamma$.

Clearly, $\gamma$ is a $W$-regular curve with $\gamma(0)=x$ and $\liminf \operatorname{int}_{t \rightarrow \infty} \operatorname{dist}\left(\gamma(t), X^{c}\right)=0$. From the hypothesis it follows $|\gamma| \cap K \neq \emptyset$ contradicting $Z \subseteq X \backslash K$.

We now give a characterization of when $d_{X}$ satisfies the minimum principle in every affine subspace parallel to a given subspace $W$ in terms of $W$-regular curves.

Lemma 4. Let $X \subseteq \mathbb{R}^{n}$ be open and let $\{0\} \neq W \subseteq \mathbb{R}^{n}$ be a subspace. Then the following are equivalent.

i) The boundary distance

$$
d_{X}: X \rightarrow \mathbb{R}, x \mapsto \operatorname{dist}\left(x, X^{c}\right)
$$

satisfies the minimum principle in $x+W$ for each $x \in X$.

ii) For each compact subset $K \subseteq X$ and every

$$
x \in\left\{y \in X ; d_{X}(y)<\operatorname{dist}\left(K, X^{c}\right)\right\}
$$

there is a $W$-regular curve $\gamma:[0, \infty) \rightarrow X$ with $\gamma(0)=x$ and $|\gamma| \cap K=\emptyset$ such that $\liminf \operatorname{in}_{t \rightarrow \infty} \operatorname{dist}\left(\gamma(t), \partial_{\infty} X\right)=0$.

Proof. We first show that ii) implies i). So let $K \subseteq(x+W) \cap X$ be compact for some $x \in X$. Assume that there is $x_{0} \in K$ with $d_{X}\left(x_{0}\right)<\operatorname{dist}\left(\partial_{x+W} K, X^{c}\right)$. Applying ii) to the compact subset $\partial_{x+W} K$ of $X$ there is a $W$-regular curve $\gamma:[0, \infty) \rightarrow X$ with $\gamma(0)=x_{0}, \liminf _{t \rightarrow \infty} \operatorname{dist}\left(\gamma(t), \partial_{\infty} X\right)=0$ and $|\gamma| \cap \partial_{x+W} K=\emptyset$. Because $\gamma$ is $W$-regular and because $x_{0} \in K \subseteq x+W$ it follows that $|\gamma| \subseteq(x+W) \cap X$. Since $\gamma(0)=x_{0}$ is contained in $\operatorname{int}_{x+W}(K)$, the interior of $K$ with respect to $x+W$, and since $\liminf \operatorname{int}_{t \rightarrow \infty} \operatorname{dist}\left(\gamma(t), \partial_{\infty} X\right)=0$ it follows from the compactness of $K \subseteq X$ that $|\gamma| \cap \partial_{x+W} K \neq \emptyset$ which gives a contradiction. 
In order to show that i) implies ii), assume that for some compact $K \subseteq X$ there is $x_{0} \in\left\{y \in X ; d_{X}(y)<\operatorname{dist}\left(K, X^{c}\right)\right\}$ such that every $W$-regular curve $\gamma:[0, \infty) \rightarrow X$ with $\gamma(0)=x_{0}$ and $\liminf _{t \rightarrow \infty} \operatorname{dist}\left(\gamma(t), \partial_{\infty} X\right)=0$ intersects $K$.

Let $Z$ be the connected component of $\left(x_{0}+W\right) \cap(X \backslash K)$ containing $x_{0}$. From the assumption on $x_{0}$ and proposition 3 it follows that $Z$ is a bounded subset of $x_{0}+W$ and

$$
\partial_{x_{0}+W} Z \subseteq K
$$

Let us denote the closure of $Z$ in $x_{0}+W$ by $C$. Then $C$ is a compact subset of $\left(x_{0}+W\right) \cap X$ by proposition 3 . By i) we obtain

$$
\min _{x \in C} d_{X}(x)=\min _{x \in \partial_{x_{0}+W} C} d_{X}(x) .
$$

Because of $x_{0} \in C$ this yields

$$
d_{X}\left(x_{0}\right) \geq \min _{x \in \partial_{x_{0}+W} C} d_{X}(x)=\operatorname{dist}\left(\partial_{x_{0}+W} Z, X^{c}\right) \geq \operatorname{dist}\left(K, X^{c}\right)
$$

where we used (2) in the last inequality. But this contradicts $d_{X}\left(x_{0}\right)<\operatorname{dist}\left(K, X^{c}\right)$.

Proof of Theorem 1. In the sequel we denote the convex hull of $x, y \in \mathbb{R}^{n}$ by $[x, y]$. In order to prove i) we fix $u \in \mathscr{E}^{\prime}(X)$ and set $K:=\operatorname{supp} \check{P}(D) u$, where as usual $\check{P}(\xi)=P(-\xi)$. Moreover, we fix $x \in\left\{y \in X ; \operatorname{dist}\left(y, X^{c}\right)<\operatorname{dist}\left(K, X^{c}\right)\right\}$. Because $d_{X}$ satisfies the minimum principle in $y+W$ for every $y \in X$ it follows from lemma 4 that there is a $W$-regular curve $\gamma:[0, \infty) \rightarrow X$ such that $\gamma(0)=x$, $|\gamma| \cap K=\emptyset$, and $\liminf _{t \rightarrow \infty} \operatorname{dist}\left(\gamma(t), X^{c}\right)=0$.

Because $\operatorname{supp} u$ is a compact subset of $X$ it follows from the properties of $\gamma$ that there is $T>0$ with $\gamma(T) \notin \operatorname{supp} u$. Moreover, we can find $\varepsilon>0$ such that the open ball $B(\gamma(T), \varepsilon)$ of radius $\varepsilon$ about $\gamma(T)$ does not intersect supp $u$, $\gamma([0, T])+B(0, \varepsilon) \subseteq X$ and $K \cap(\gamma([0, T])+B(0, \varepsilon))=\emptyset$, where $\gamma([0, T])+B(0, \varepsilon)=$ $\{y+z ; y \in \gamma([0, T]), z \in B(0, \varepsilon)\}$.

Next, we choose $0=t_{0}<t_{1}<\ldots<t_{k}=T$ such that for each $j=1, \ldots, k$ the restriction of $\gamma$ to $\left[t_{j-1}, t_{j}\right]$ is continuously differentiable and

$$
\left|\int_{t_{j-1}}^{t_{j}} \gamma^{\prime}(t) d t\right|<\frac{\varepsilon}{2}
$$

We define

$$
f:[0, k] \rightarrow \mathbb{R}^{n}, s \mapsto \gamma\left(t_{\lfloor s\rfloor}\right)+(s-\lfloor s\rfloor) \int_{t_{\lfloor s\rfloor}}^{t_{\lfloor s\rfloor}+1} \gamma^{\prime}(t) d t,
$$

where $\lfloor s\rfloor$ denotes the integer part of $s$. Then $f$ is a polygonal curve in $x+W$ by the $W$-regularity of $\gamma$ with $f([j-1, j])=\left[\gamma\left(t_{j-1}\right), \gamma\left(t_{j}\right)\right], j=1, \ldots, k$. Moreover, due to the choice of $\varepsilon$, we have $|f|+B\left(0, \frac{\varepsilon}{2}\right) \subseteq X \backslash K$.

For $N \in\left\{y \in \mathbb{R}^{n} ; P_{m}(y)=0\right\} \backslash\{0\}$ and $\alpha \in \mathbb{R}$ let

$$
H_{N, \alpha}=\left\{x \in \mathbb{R}^{n} ;\langle x, N\rangle=\alpha\right\}
$$

be the corresponding characteristic hyperplane. Since $\left[\gamma\left(t_{k-1}\right), \gamma(T)\right] \subseteq x+W$ and $N \in W^{\perp}$ it follows that $H_{N, \alpha}$ intersects $B(\gamma(T), \varepsilon)$ whenever $H_{N, \alpha}$ intersects $\left[\gamma\left(t_{k-1}\right), \gamma(T)\right]+B(0, \varepsilon)$. By the choice of $\varepsilon$ we have $u_{\mid B(\gamma(T), \varepsilon)}=0$ so that by $[10$, Theorem 8.6.8, vol. I] $u$ vanishes in $\left[\gamma\left(t_{k-1}\right), \gamma(T)\right]+B(0, \varepsilon)$.

Iteration of the above argument yields that $u$ vanishes in $|f|+B(0, \varepsilon)$, in particular $x=\gamma(0)=f(0)$ does not belong to $\operatorname{supp} u$. As $x$ was an arbitrary point with $\operatorname{dist}\left(x, X^{c}\right)<\operatorname{dist}\left(K, X^{c}\right)$ it follows from the definition of $K$ that

$$
\operatorname{dist}\left(\operatorname{supp} u, X^{c}\right) \geq \operatorname{dist}\left(K, X^{c}\right)=\operatorname{dist}\left(\operatorname{supp} \check{P}(D), X^{c}\right) \text {. }
$$


Since trivially $\operatorname{dist}\left(\operatorname{supp} u, X^{c}\right) \leq \operatorname{dist}\left(\operatorname{supp} \check{P}(D) u, X^{c}\right)$ it follows from [10, Theorem 10.6.3, vol. II] that $X$ is $P$-convex for supports.

In order to prove ii), we replace in the above arguments supp by sing supp, $N \in\left\{y \in \mathbb{R}^{n} ; P_{m}(y)=0\right\}$ by $N \in\left\{y \in \mathbb{R}^{n} ;\left(\sigma_{\check{P}}(y)=\right) \sigma_{P}(y)=0\right\}$, and the reference to [10, Theorem 10.6.3, vol. II] by [5, Corollary 2].

As a corollary to Theorem 1 we obtain the next result characterizing $P$-convexity for supports for particular polynomials $P$. This characterization is in particular applicable to the the Schrödinger operator $P(D)=\Delta_{x}+i \frac{\partial}{\partial t}$ and the heat operator $P(D)=\Delta_{x}-\frac{\partial}{\partial t}$, or more generally, to non-degenerate parabolic operators.

Corollary 5. Let $X \subseteq \mathbb{R}^{n}$ be open and let $P$ be a polynomial with principal part $P_{m}$ such that $\left\{x \in \mathbb{R}^{n} ; P_{m}(x)=0\right\}$ is a one-dimensional subspace of $\mathbb{R}^{n}$. Then, the following are equivalent.

i) $P(D)$ is surjective on $C^{\infty}(X)$.

ii) $d_{X}$ satisfies the minimum principle in every characteristic hyperplane.

Proof. We set $W:=\left\{x \in \mathbb{R}^{n} ; P_{m}(x)=0\right\}^{\perp}$. That ii) implies i) follows from Theorem 1 while the reverse implication is [10, Theorem 10.8.1, vol. II].

The analogous result for $P$-convexity for singular supports is the following.

Corollary 6. Let $P \neq 0$ be a polynomial such that $\left\{x \in \mathbb{R}^{n} ; \sigma_{P}(x)=0\right\}=W^{\perp}$ for some subspace $W \subseteq \mathbb{R}^{n}$ with $\sigma_{P}\left(W^{\perp}\right)=0$. Then, for $X \subseteq \mathbb{R}^{n}$ the following are equivalent.

i) $X$ is $P$-convex for singular supports.

ii) $d_{X}$ satisfies the minimum principle in $x+W$ for every $x \in \mathbb{R}^{n}$.

Proof. That ii) implies i) follows again from Theorem 1 while the reverse implication is [10, Corollary 11.3.2, vol. II].

Remark 7. We do not know if the condition " $\sigma_{P}\left(W^{\perp}\right)=0$ " in Corollary 6 is redundant.

Recall that a polynomial $P \in \mathbb{C}\left[X_{1}, \ldots, X_{d}\right]$ is said to act along a subspace $W$ of $\mathbb{R}^{d}$ if $P(x)=P\left(\pi_{W}(x)\right)$ for every $x \in \mathbb{R}^{d}$, where $\pi_{W}$ denotes the orthogonal projection of $\mathbb{R}^{d}$ onto $W$. It is easily seen that $P$ acts along a subspace $W$ of $\mathbb{R}^{d}$ if and only if $P(x+y)=P(x)$ holds for every $x \in \mathbb{R}^{d}$ and each $y \in W^{\perp}$. Moreover, a polynomial $P$ acting along a subspace $W$ is said to be elliptic on $W$ if $P_{m}(x) \neq 0$ for all $x \in W \backslash\{0\}$.

Lemma 8. Let $P \in \mathbb{C}\left[X_{1}, \ldots, X_{n}\right]$ be a non-constant polynomial which acts along a subspace $W \subseteq \mathbb{R}^{n}$ and is elliptic as a polynomial on $W$. Then $\sigma_{P}(V)=0$ holds for a subspace $V \subseteq \mathbb{R}^{n}$ if and only if $V \subseteq W^{\perp}$.

Proof. Without loss of generality we may assume $W=\mathbb{R}^{k} \times\{0\}$ with $1 \leq k \leq n$ and $0 \in \mathbb{R}^{n-k}$. For $x=\left(x_{1}, \ldots, x_{n}\right)$ we denote $x^{\prime}=\left(x_{1}, \ldots, x_{k}\right)$.

Clearly, $V \subseteq W^{\perp}$ implies $\sigma_{P}(V)=0$. On the other hand, if $V$ is not a subspace of $W^{\perp}$ there is $x \in V$ with $\left|x^{\prime}\right|=1$. From the ellipticity of $P$ on $\mathbb{R}^{k}$ it follows that for some $C>0$ we have

$$
\forall \xi \in \mathbb{R}^{n}: C\left|\xi^{\prime}\right|^{m} \leq\left|P\left(\xi^{\prime}\right)\right|
$$


where $m \in \mathbb{N}_{0}$ is the degree of $P$. Thus, for $\xi \in \mathbb{R}^{n}$ and $t>0$ we have

$$
\begin{aligned}
\tilde{P}_{\operatorname{span}\{x\}}(\xi, t)^{2} & =\sup _{|\theta| \leq t}\left|P\left(\xi^{\prime}+\theta x^{\prime}\right)\right|^{2} \\
& \geq C \sup _{|\theta| \leq t}\left|\xi^{\prime}+\theta x^{\prime}\right|^{2 m} \\
& \geq \frac{C}{2}\left(\left|\xi^{\prime}+t x^{\prime}\right|^{2 m}+\left|\xi^{\prime}-t x^{\prime}\right|^{2 m}\right) \\
& =\frac{C}{2}\left(\left(\sum_{j=1}^{k}\left(\xi_{j}+t x_{j}\right)^{2}\right)^{m}+\left(\sum_{j=1}^{k}\left(\xi_{j}-t x_{j}\right)^{2}\right)^{m}\right) \\
& \geq \frac{C}{2}\left(\sum_{j=1}^{k}\left(\left(\xi_{j}+t x_{j}\right)^{2 m}+\left(\xi_{j}-t x_{j}\right)^{2 m}\right)\right) \\
& \geq C\left(\sum_{j=1}^{k} \xi_{j}^{2 m}+t^{2 m} \sum_{j=1}^{k} x_{j}^{2 m}\right) .
\end{aligned}
$$

Moreover, for suitable $C^{\prime}, D>0$ we have for all $\xi \in \mathbb{R}^{n}$ and $t>0$

$$
\tilde{P}(\xi, t)^{2} \leq C^{\prime}\left(t^{2 m}+\left|\xi^{\prime}\right|^{2 m}\right) \leq D\left(t^{2 m}+\sum_{j=1}^{k} \xi_{j}^{2 m}\right) .
$$

If we set $\alpha:=\sum_{j=1}^{k} x_{j}^{2 m}$ and take into account that $\alpha>0$ because of $\left|x^{\prime}\right|=1$ it follows that for every $\xi \in \mathbb{R}^{n}$ and $t>0$ we have

$$
\frac{\tilde{P}_{\operatorname{span}\{x\}}(\xi, t)^{2}}{\tilde{P}(\xi, t)^{2}} \geq \frac{C\left(\sum_{j=1}^{k} \xi_{j}^{2 m}+t^{2 m} \alpha\right)}{D\left(\sum_{j=1}^{k} \xi_{j}^{2 m}+t^{2 m}\right)} \geq \frac{C}{D} \alpha>0,
$$

so that

$$
\sigma_{P}(V) \geq \sigma_{P}(x) \geq \frac{C}{D} \alpha>0
$$

which proves the lemma.

The next theorem complements a result of Nakane [18] mentioned in the introduction.

Theorem 9. Let $P \in \mathbb{C}\left[X_{1}, \ldots, X_{n}\right]$ be a polynomial which acts along a subspace $W \subseteq \mathbb{R}^{n}$ and is elliptic as a polynomial on $W$. Then, for an open subset $X \subseteq \mathbb{R}^{n}$ the following are equivalent.

i) $X$ is $P$-convex for supports.

ii) $X$ is $P$-convex for singular supports.

iii) $d_{X}$ satisfies the minimum principle in $x+W$ for every $x \in \mathbb{R}^{n}$.

In particular, $P(D)$ is surjective on $C^{\infty}(X)$ if and only if $P(D)$ is surjective on $\mathscr{D}^{\prime}(X)$.

Proof. That i) and iii) are equivalent is [10, Theorem 10.8.5] while the equivalence of ii) and iii) follows from Corollary 6 and Lemma 8.

Corollary 10. Let $P(x)=\alpha \prod_{j=1}^{l}\left(\left\langle N_{j}, x\right\rangle+c_{j}\right)$ where $N_{j} \in \mathbb{C}^{n} \backslash\{0\}, \alpha, c_{j} \in \mathbb{C}, j=$ $1, \ldots, l$, . Then for an open subset $X \subseteq \mathbb{R}^{n}$ the following are equivalent.

i) $X$ is $P$-convex for supports.

ii) $X$ is $P$-convex for singular supports.

iii) $d_{X}$ satisfies the minimum principle in $x+W$ for every $x \in \mathbb{R}^{n}$, where $W$ is one of the subspaces span $\left\{\right.$ Re $\left.N_{j}, \operatorname{Im} N_{j}\right\}, j=1 \ldots, l$. 
Proof. Since each of the the first order operators $\left\langle N_{j}, D\right\rangle+c_{j}$ acts along one of the subspaces $\operatorname{span}\left\{\operatorname{Re} N_{j}, \operatorname{Im} N_{j}\right\}$ and is elliptic there, the corollary follows directly from Theorem 9 and the fact that these operators commute.

\section{SurJectivity of CERTAin AUgmented DifFEREntial Operators}

In this section we show that for certain partial differential operators, including non-degenerate parabolic operators like the heat operator, surjectivity of $P(D)$ on $\mathscr{D}^{\prime}(X)$ implies the surjectivity of the augmented operator $P^{+}(D)$ on $\mathscr{D}^{\prime}(X \times \mathbb{R})$, where $P^{+}\left(x_{1}, \ldots, x_{n}, x_{n+1}\right):=P\left(x_{1}, \ldots, x_{n}\right)$. It was shown in [12], that in case of $n=2$ the augmented operator of a surjective partial differential operator $P(D)$ is always surjective while in [13] for $n \geq 3$ a hypoelliptic differential operator $P(D)$ was constructed for which there is some open $X \subseteq \mathbb{R}^{n}$ such that $P(D)$ is surjective on $\mathscr{D}^{\prime}(X)$ while the augmented operator $P^{+}(D)$ is not surjective on $\mathscr{D}^{\prime}(X \times \mathbb{R})$. Thus, although not true in general, for certain differential operators, including the heat operator, the problem of parameter dependence for solutions of partial differential equations [1, Problem 9.1] has a positive solution, as will be shown as a consequence of the results from this section in section 4 .

Apart from the functional $\sigma_{P}$ we also use

$$
\sigma_{P}^{0}(V):=\inf _{t \geq 1, \xi \in \mathbb{R}^{n}} \frac{\tilde{P}_{V}(\xi, t)}{\tilde{P}(\xi, t)},
$$

where $V \subseteq \mathbb{R}^{n}$ is again a subspace. Moreover, we set $\sigma_{p}^{0}(y):=\sigma_{P}^{0}(\operatorname{span}\{y\})$ for $y \in \mathbb{R}^{n}$. Furthermore, we define

$$
\pi: \mathbb{R}^{n+1} \rightarrow \mathbb{R}^{n},\left(x_{1}, \ldots, x_{n+1}\right) \mapsto\left(x_{1}, \ldots, x_{n}\right)
$$

and we set $A^{\prime}:=\pi(A)$ for $A \subseteq \mathbb{R}^{n+1}$, respectively $x^{\prime}:=\pi(x)$ for $x \in \mathbb{R}^{n+1}$. The reason for using $\sigma_{P}^{0}$ is the following lemma. For its proof see [5, Lemma 1].

Lemma 11. For a polynomial $P \in \mathbb{C}\left[X_{1}, \ldots, X_{n}\right]$ and a subspace $V \subseteq \mathbb{R}^{n+1}$ the following hold.

i) $\sigma_{P^{+}}\left(V^{\prime} \times \mathbb{R}\right)=\sigma_{P^{+}}\left(V^{\prime} \times\{0\}\right)=\sigma_{P}^{0}\left(V^{\prime}\right)$.

ii) $\sigma_{P+}(V)=0$ if and only if $\sigma_{P}^{0}\left(V^{\prime}\right)=0$.

We continue with an easy geometrical observation. For its proof we need the following trivial proposition.

Proposition 12. Let $X$ and $Y$ be Hausdorff topological spaces, $f: X \rightarrow Y$ continuous and open, and let $K \subseteq X$ be compact. Then $\partial f(K) \subseteq f(\partial K)$.

Proof. Let $y \in \partial f(K)$ and $x \in K$ with $f(x)=y$. Suppose that $x \notin \partial K$. Then there is an open neighborhood $V$ of $x$ such that $V \subseteq K$. Since $f$ is open it follows that $f(V)$ is an open neighborhood of $y=f(x)$ which is contained in $f(K)$ contradicting $y \in \partial f(K)$.

Proposition 13. Let $X \subseteq \mathbb{R}^{n}$ be open and $F \subseteq \mathbb{R}^{n+1}$ be closed. If $d_{X}$ satisfies the minimum principle in $F^{\prime}$ then $d_{X \times \mathbb{R}}$ satisfies the minimum principle in $F$. Moreover, if $F$ is an affine subspace such that $\left\{x_{n+1} ; x \in F\right\}$ is bounded and $d_{X \times \mathbb{R}}$ satisfies the minimum principle in $F$ then $d_{X}$ satisfies the minimum principle in $F^{\prime}$.

Proof. Let $d_{X}$ satisfies the minimum principle in $F^{\prime}$. Let $K \subseteq F \cap(X \times \mathbb{R})$ be compact. We assume that there is $x_{0} \in K$ such that

$$
d_{X \times \mathbb{R}}\left(x_{0}\right)<\min _{x \in \partial_{F} K} d_{X \times \mathbb{R}}(x) .
$$


Since $d_{X \times \mathbb{R}}(x)=d_{X}\left(x^{\prime}\right)$ it follows

$$
\begin{aligned}
d_{X}\left(x_{0}^{\prime}\right) & =d_{X \times \mathbb{R}}\left(x_{0}\right)<\min _{x \in \partial_{F} K} d_{X \times \mathbb{R}}(x) \\
& =\min \left\{d_{X}\left(x^{\prime}\right) ; x^{\prime} \in \pi_{\mid F}\left(\partial_{F} K\right)\right\} .
\end{aligned}
$$

Applying Proposition 12 to $\pi_{\mid F} F \rightarrow F^{\prime}$ we obtain $\pi_{\mid F}\left(\partial_{F} K\right) \supseteq \partial_{F^{\prime}} K^{\prime}$ so that the previous inequality yields

$$
d_{X}\left(x_{0}^{\prime}\right)<\min _{\partial_{F^{\prime}} K^{\prime}} d_{X}\left(x^{\prime}\right)
$$

Since $x_{0}^{\prime} \in K^{\prime}$ and since $d_{X}$ satisfies the minimum principle this gives a contradiction. Thus

$$
d_{X \times \mathbb{R}}\left(x_{0}\right) \geq \min _{x \in \partial_{F} K} d_{X \times \mathbb{R}}(x)
$$

for every $x_{0} \in K$ so that $d_{X \times \mathbb{R}}$ satisfies the minimum principle in $F$.

Now assume that $F$ is an affine subspace such that $\left\{x_{n+1} ; x \in F\right\}$ is bounded and that $d_{X \times \mathbb{R}}$ satisfies the minimum principle in $F$. Since $\left\{x_{n+1} ; x \in F\right\}$ is bounded the subspace $F-F$ of $\mathbb{R}^{n+1}$ is contained in $\mathbb{R}^{n} \times\{0\}$. It follows for $x, y \in F$ with $\pi(x)=\pi(y)$ that $x^{\prime}=y^{\prime}$ and because of $x-y \in F-F \subseteq \mathbb{R}^{n} \times\{0\}$ we obtain $x=y$. Therefore, $\pi_{\mid F}$ is injective so that

$$
\pi_{\mid F}: F \rightarrow F^{\prime}
$$

is a homeomorphism.

Let $K \subseteq F^{\prime} \cap X$ be compact. It follows that $K \times \mathbb{R} \subseteq\left(F^{\prime} \times \mathbb{R}\right) \cap(X \times \mathbb{R})$, thus $(K \times \mathbb{R}) \cap F \subseteq F \cap(X \times \mathbb{R})$. Because $\left\{x_{n+1} ; x \in F\right\}$ is bounded

$$
L:=(K \times \mathbb{R}) \cap F
$$

is a compact subset of $F \cap(X \times \mathbb{R})$ with $\pi(L)=K$. Therefore, and since $d_{X \times \mathbb{R}}$ satisfies the minimum principle in $F$ we have

$$
\begin{aligned}
\min _{x^{\prime} \in K} d_{X}\left(x^{\prime}\right) & =\min \left\{d_{X}\left(x^{\prime}\right) ; x^{\prime} \in \pi(L)\right\}=\min \left\{d_{X}(\pi(x)) ; x \in L\right\} \\
& =\min _{x \in L} d_{X \times \mathbb{R}}(x)=\min _{x \in \partial_{F} L} d_{X \times \mathbb{R}}(x) \\
& =\min \left\{d_{X}\left(x^{\prime}\right) ; x^{\prime} \in \pi_{\mid F}\left(\partial_{F} L\right)\right\} \\
& =\min \left\{d_{X}\left(x^{\prime}\right) ; x^{\prime} \in \partial_{F}^{\prime} K\right\}=\min _{x^{\prime} \in \partial_{F}^{\prime} K} d_{X}\left(x^{\prime}\right)
\end{aligned}
$$

where we used that $\pi_{\mid F}$ is a homeomorphism onto $F^{\prime}$ and $\pi(L)=K$ in the last step.

The next proposition will be useful in the proof of the following theorem.

Proposition 14. Let $P$ be a polynomial and let $V \subseteq \mathbb{R}^{n}$ be a subspace such that $P$ is constant on $V$. Then

$$
\forall x \in \mathbb{R}^{n}, \xi \in V: P(x+\xi)=P(x),
$$

i.e. $P$ acts along $V^{\perp}$.

Proof. By an appropriate linear change of coordinates we may assume without loss of generality that $V=\mathbb{R}^{k} \times\{0\}^{n-k}$ with $k=\operatorname{dim} V$. Since $P$ is constant on $V$ it follows

$$
\forall \xi \in V, \alpha \in \mathbb{N}_{0}^{n} \cap V, \alpha \neq 0: \partial^{\alpha} P(\xi)=0 .
$$

Writing $\alpha \in \mathbb{N}_{0}^{n}$ as $\alpha=\alpha^{\prime}+\alpha^{\prime \prime}$ with $\alpha^{\prime} \in \mathbb{N}_{0}^{n} \cap V$ and $\alpha^{\prime \prime} \in \mathbb{N}_{0}^{n} \cap V^{\perp}$ it follows

$$
\forall \xi \in V, \alpha \in \mathbb{N}_{0}^{n}, \alpha^{\prime} \neq 0: \partial^{\alpha} P(\xi)=\partial^{\alpha^{\prime \prime}} \partial^{\alpha^{\prime}} P(\xi)=0 .
$$


Since $\xi_{k+1}=\ldots=\xi_{n}=0$ for every $\xi \in V$ this implies together with Taylor's Theorem

$$
\begin{aligned}
P(x+\xi) & =\sum_{\alpha \in \mathbb{N}_{0}^{n}} \frac{\partial^{\alpha} P(0)}{\alpha !}(x+\xi)^{\alpha}=\sum_{\alpha \in \mathbb{N}_{0}^{n} \cap V^{\perp}} \frac{\partial^{\alpha} P(0)}{\alpha !}(x+\xi)^{\alpha} \\
& =\sum_{\alpha \in \mathbb{N}_{0}^{n} \cap V^{\perp}} \frac{\partial^{\alpha} P(0)}{\alpha !} x^{\alpha}=P(x)
\end{aligned}
$$

for every $x \in \mathbb{R}^{n}$ and $\xi \in V$.

Recall that a polynomial $P$ is called semi-elliptic if it is possible to write

$$
P(\xi)=\sum_{|\alpha: \mathbf{m}| \leq 1} c_{\alpha} \xi^{\alpha}
$$

such that

$$
\forall \xi \in \mathbb{R}^{n} \backslash\{0\}: \sum_{|\alpha: \mathbf{m}|=1} c_{\alpha} \xi^{\alpha} \neq 0,
$$

where $\mathbf{m} \in \mathbb{N}_{0}^{n}$ and $|\alpha: \mathbf{m}|=\sum_{j=1}^{n} \alpha_{j} / m_{j}$. With $m_{1}=1$ and $m_{j}=2$ for $j>1$ it follows that the polynomial inducing the heat operator is semi-elliptic.

Theorem 15. Let $P \in \mathbb{C}\left[X_{1}, \ldots, X_{n}\right]$ be a semi-elliptic polynomial with principal part $P_{m}$ and let $X \subseteq \mathbb{R}^{n}$ be open. Then the following are equivalent.

i) $X \times \mathbb{R}$ is $P^{+}$-convex for singular supports.

ii) $X$ is $P_{m}$-convex for supports.

iii) $X$ is $P_{m}$-convex for singular supports.

iv) $d_{X}$ satisfies the minimum principle in $x+\left\{y \in \mathbb{R}^{n} ; P_{m}(y)=0\right\}^{\perp}$ for every $x \in \mathbb{R}^{n}$

Proof. It is shown in [5, Proposition 2, Lemma 3] that $Z:=\left\{x \in \mathbb{R}^{n} ; P_{m}(x)=0\right\}$ is a subspace of $\mathbb{R}^{n}$ and that $\sigma_{P^{+}}(V)=0$ if and only if $V$ is a subspace of $Z \times \mathbb{R}$. In particular,

$$
\left\{x \in \mathbb{R}^{n+1} ; \sigma_{P^{+}}(x)=0\right\}=Z \times \mathbb{R}=\left(Z^{\perp} \times\{0\}\right)^{\perp} .
$$

Since $\sigma_{P^{+}}(Z \times \mathbb{R})=0$ by Lemma 11 it follows from [10, Corollary 11.3.2] that $d_{X \times \mathbb{R}}$ satisfies the minimum principle in $x+(Z \times \mathbb{R})^{\perp}$ for every $x \in \mathbb{R}^{n+1}$ if $X \times \mathbb{R}$ is $P^{+}$-convex for singular supports. Proposition 13 therefore implies that $d_{X}$ satisfies the minimum principle in every affine subspace parallel to $Z^{\perp}$ if $X \times \mathbb{R}$ is $P^{+}$-convex for singular supports. Thus, i) implies iv).

On the other hand, if $d_{X}$ satisfies the minimum principle in every affine subspace parallel to $Z^{\perp}$ it follows from Proposition 13 that $d_{X \times \mathbb{R}}$ satisfies the minimum principle in every affine subspace parallel to $(Z \times \mathbb{R})^{\perp}=Z^{\perp} \times\{0\}$. From (3) together with Theorem 1 we therefore conclude that $X \times \mathbb{R}$ is $P^{+}$-convex for singular supports. Hence, iv) implies i).

In order to prove the remaining equivalences, we observe that by Proposition $14 P_{m}$ is a polynomial acting along the subspace $Z^{\perp}$. Since $Z \cap Z^{\perp}=\{0\} P_{m}$ is elliptic as a polynomial on $Z^{\perp}$ so that Theorem 9 yields the equvalences of ii), iii), and iv).

We are now able to prove the main result of this section. Part iii) is in particular applicable to non-degenerate parabolic operators like the heat operator.

Theorem 16. Let $P$ be a polynomial with principal part $P_{m}$ and let $X \subseteq \mathbb{R}^{n}$ be open such that $P(D): \mathscr{D}^{\prime}(X) \rightarrow \mathscr{D}^{\prime}(X)$ is surjective. Then the augmented operator $P^{+}(D): \mathscr{D}^{\prime}(X \times \mathbb{R}) \rightarrow \mathscr{D}^{\prime}(X \times \mathbb{R})$ is surjective if

i) $P$ acts along a subspace $W$ and is elliptic as a polynomial on $W$, or 
ii) $P(x)=\alpha \prod_{j=1}^{l}\left(\left\langle N_{j}, x\right\rangle+c_{j}\right)$ where $N_{j} \in \mathbb{C}^{n} \backslash\{0\}$ and $\alpha, c_{j} \in \mathbb{C}, j=1 \ldots, l$, or

iii) $P$ is a semi-elliptic polynomial for which $\left\{x \in \mathbb{R}^{n} ; P_{m}(x)=0\right\}$ is a onedimensional subspace.

Proof. Since $X \times \mathbb{R}$ is $P^{+}$-convex for supports whenever $X$ is $P$-convex for supports by $\left[5\right.$, Proposition 1] we only have to show that $X \times \mathbb{R}$ is $P^{+}$-convex for singular supports.

If $P$ acts along a subspace and is elliptic there, the same holds for $P^{+}$. As $X \times \mathbb{R}$ is $P^{+}$-convex for supports it follows from Theorem 9 that $X \times \mathbb{R}$ is also $P^{+}$-convex for singular supports, proving i).

For $P(x)=\alpha \prod_{j=1}^{l}\left(\left\langle N_{j}, x\right\rangle+c_{j}\right)$, each of the factors of $P$ acts along a subspace and is elliptic there. Thus applying i) to each of the factors yields ii).

In case of iii) it follows from [10, Theorem 10.8.1] that $d_{X}$ satisfies the minimum principle in every characteristic hyperplane. Thus, it follows from Theorem 15 that $X \times \mathbb{R}$ is $P^{+}$-convex for singular supports.

Remark 17. a) It was kindly pointed out to us by D. Vogt that for $P(D)=$ $\langle D, N\rangle+c$ in case of $N \in \mathbb{R}^{n}$ the surjectivity of $P(D)$ on $\mathscr{D}^{\prime}(X)$ already implies the existence of a continuous linear right inverse of $P(D)$ on $\mathscr{D}^{\prime}(X)$ (see [15]) and thus a continuous linear right inverse of $P^{+}(D)$ on $\mathscr{D}^{\prime}(X \times \mathbb{R})$. In particular, $P^{+}(D)$ is then surjective on $\mathscr{D}^{\prime}(X \times \mathbb{R})$. However, due to the well-known result of Grothendieck that elliptic $P(D)$ do not have continuous linear right inverses for $n \geq 2$ (see e.g. [24, Theorem C.1]) on $C^{\infty}(X)$ combined with [15, Theorem 2.7] the Cauchy-Riemann operator $\partial_{\bar{z}}$ shows that on $\mathscr{D}^{\prime}(X)$ a surjective first order operator $P(D)=\langle D, N\rangle+c$ with $N \in \mathbb{C}^{n} \backslash \mathbb{R}^{n}$ in general does not have a continuous linear right inverse.

b) As shown in the proof of Theorem 15, the principal part $P_{m}$ of a semi-elliptic polynomial $P$ acts along the subspace $\left\{x \in \mathbb{R}^{n} ; P_{m}(x)=0\right\}^{\perp}$ and is elliptic as a polynomial on this subspace. Therefore, applying Theorem 16, Theorem 9, and Proposition 13, it follows that for a semi-elliptic polynomial the statement

v) $X \times \mathbb{R}$ is $P_{m}^{+}$-convex for singular supports.

can be added to the equivalences in Theorem 15.

\section{Surjectivity of Differential operators on VECTOR-VALUED FUNCTIONS AND DISTRIBUTIONS AND LINEAR TOPOLOGICAL INVARIANTS}

In this section we consider the problem whether surjectivity of a differential operator $P(D)$ on $C^{\infty}(X)$, resp. on $\mathscr{D}^{\prime}(X)$, passes on to surjectivity of $P(D)$ on spaces of vector-valued functions, resp. vector-valued distributions. As usual, for a locally convex space $E$ we denote by $C^{\infty}(X, E)$ the space of smooth functions with values in $E$ and by $\mathscr{D}^{\prime}(X, E)$ the space of $E$-valued distributions, i.e. the space of continuous linear operators from $\mathscr{D}(X)$ to $E$ equipped with its usual locally convex topology. As explained in the introduction, surjectivity of $P(D)$ on spaces of vector-valued smooth functions and distributions is connected with the problem of parameter dependence for solutions of the equation $P(D) u_{\lambda}=f_{\lambda}$ on the corresponding scalar-valued spaces. Obviously, for two topologically isomorphic locally convex spaces $E_{1}$ and $E_{2}, P(D)$ is surjective on $C^{\infty}\left(X, E_{1}\right)$ (resp. $\mathscr{D}^{\prime}\left(X, E_{1}\right)$ ) if and only if $P(D)$ is surjective on $C^{\infty}\left(X, E_{2}\right)$ (resp. $\mathscr{D}^{\prime}\left(X, E_{2}\right)$ ). As described in the introduction, this problem is addressed by proving that the kernels $C_{P}^{\infty}(X)$, resp. $\mathscr{D}_{P}^{\prime}(X)$ have properties $(\Omega)$, resp. $(P \Omega)$ when $P(D)$ is surjective on $C^{\infty}(X)$, resp. $\mathscr{D}^{\prime}(X)$. 
Theorem 18. Let $X \subseteq \mathbb{R}^{n}$ be open and let $P$ be a polynomial with principle part $P_{m}$.

a) Let $P$ be semi-elliptic such that $\left\{x \in \mathbb{R}^{n} ; P_{m}(x)=0\right\}$ is a one-dimensional subspace of $\mathbb{R}^{n}$. If $X$ is $P$-convex for supports then $C_{P}^{\infty}(X)$ has property $(\Omega)$.

b) Let $P$ act along a subspace of $\mathbb{R}^{n}$ such that it is elliptic there. If $P(D)$ is surjective on $\mathscr{D}^{\prime}(X)$ then $\mathscr{D}_{P}^{\prime}(X)$ has property $(P \Omega)$.

c) Let $P(x)=\alpha \prod_{j=1}^{l}\left(\left\langle N_{j}, x\right\rangle-c_{j}\right)$ where $\alpha, c_{j} \in \mathbb{C}$ and $N_{j} \in \mathbb{C}^{n} \backslash\{0\}, j=$ $1, \ldots, l$ and let $P(D)$ be surjective on $\mathscr{D}^{\prime}(X)$. Then $\mathscr{D}_{P}^{\prime}(X)$ has $(P \Omega)$.

Proof. First, let $P$ be a semi-elliptic polynomial such that $\left\{x \in \mathbb{R}^{n} ; P_{m}(x)=0\right\}$ is a one-dimensional subspace of $\mathbb{R}^{n}$. Since semi-elliptic operators are hypoelliptic it follows that $P(D)$ is surjective on $\mathscr{D}^{\prime}(X)$ if it is surjective on $C^{\infty}(X)$. Thus, by Theorem 16 it follows that $P^{+}(D)$ is surjective on $\mathscr{D}^{\prime}(X \times \mathbb{R})$ so that $\mathscr{D}_{P}^{\prime}(X)$ has $(P \Omega)$ by $[1$, Proposition 8.3$]$. Since $P(D)$ is hypoelliptic, $\mathscr{D}_{P}^{\prime}(X)$ and $C_{P}^{\infty}(X)$ coincide as locally convex spaces by [25, Theorem 52.1], thus the Fréchet-Schwartz space $C_{P}^{\infty}(X)$ has $(\Omega)$ by $[1$, Proposition 5.3$]$. This proves a).

For a polynomial $P$ which acts along a subspace and is elliptic there, using muatis mutandis the same arguments used to prove a) yield b).

Finally, let $P(x)=\alpha \prod_{j=1}^{l}\left(\left\langle N_{j}, x\right\rangle-c_{j}\right)$ and let $P(D)$ be surjective on $\mathscr{D}^{\prime}(X)$. Each factor of $P$ acts along one of the subspaces $\operatorname{span}\left\{\operatorname{Re} N_{j}, \operatorname{Im} N_{j}\right\}$ and is elliptic there so it follows from part b) and [1, Proposition 8.3] that each of the factors of $P(D)$ and therefore $P(D)$ itself is surjective on $\mathscr{D}^{\prime}(X \times \mathbb{R})$. Another reference to $\left[1\right.$, Proposition 8.3] yields that $\mathscr{D}_{P}^{\prime}(X)$ has $(P \Omega)$.

Corollary 19. Let $X \subseteq \mathbb{R}^{n}$ be open and let $P$ be a polynomial with principle part $P_{m}$.

a) Let $F$ be a Fréchet space with property $(D N)$ and let $P$ be semi-elliptic such that $\left\{x \in \mathbb{R}^{n} ; P_{m}(x)=0\right\}$ is a one-dimensional subspace of $\mathbb{R}^{n}$ and such that $P(D)$ is surjective on $C^{\infty}(X)$. Then $P(D)$ is surjective on $C^{\infty}\left(X, F^{\prime}\right)$.

b) Let $E$ be a locally convex space such that $E$ is either the strong dual of a nuclear Fréchet space with $(D N)$, the strong dual of a Fréchet-Schwartz space with $(D N)$ which has an absolute Schauder basis, or $\mathscr{D}^{\prime}(Y)$, where $Y \subseteq \mathbb{R}^{m}$ is open.

i) Let $P$ be semi-elliptic such that $\left\{x \in \mathbb{R}^{n} ; P_{m}(x)=0\right\}$ is a onedimensional subspace of $\mathbb{R}^{n}$ and such that $P(D)$ is surjective on $C^{\infty}(X)$. Then $P(D)$ is surjective on $\mathscr{D}^{\prime}(X, E)$.

ii) Assume $P$ acts along a subspace of $\mathbb{R}^{n}$ and is elliptic there such that $P(D)$ is surjective on $\mathscr{D}^{\prime}(X)$. Then $P(D)$ is surjective on $\mathscr{D}^{\prime}(X, E)$.

iii) Let $P(x)=\alpha \prod_{j=1}^{l}\left(\left\langle N_{j}, x\right\rangle-c_{j}\right)$ where $\alpha, c_{j} \in \mathbb{C}$ and $N_{j} \in \mathbb{C}^{n} \backslash\{0\}, j=$ $1, \ldots, l$ such that $P(D)$ is surjective on $\mathscr{D}^{\prime}(X)$. Then $P(D)$ is surjective on $\mathscr{D}^{\prime}(X, E)$.

Proof. If the hypothesis of a) are satisfied it follows from part a) of Theorem 18 that $C_{P}^{\infty}(X)$ has property $(\Omega)$. If $F$ is a Fréchet space with $(D N)$ it follows from $[28$, Theorem $2.4 \mathrm{~b})]$ that $P(D)$ is surjective on $C^{\infty}\left(X, F^{\prime}\right)$ which proves a). In case $F$ is a Fréchet-Schwartz space with absolute Schauder basis, $F$ is topologically isomorphic to a Köthe sequence space $\lambda^{1}(A)$ (see [17, Proposition 27.26]). If $F$ additionally has $(D N)$ it follows from $[1$, Theorem 5.6, Corollary 4.2, and Proposition 3.3] that $P(D)$ is surjective on $\mathscr{D}^{\prime}\left(X, F^{\prime}\right)$. In case $F$ is a nuclear Fréchet space with $(D N)$ it follows again from $[1$, Theorem 5.6, Corollary 4.2, and Proposition 3.3 that $P(D)$ is surjective on $\mathscr{D}^{\prime}\left(X, F^{\prime}\right)$. Since by the Schwartz-Kernel Theorem $\mathscr{D}^{\prime}(X \times \mathbb{R})$ and $\mathscr{D}^{\prime}\left(X, \mathscr{D}^{\prime}(\mathbb{R})\right)$ are canonically isomorphic and since under this 
isomorphism $P^{+}(D)$ and $P(D)$ on $\mathscr{D}^{\prime}\left(X, \mathscr{D}^{\prime}(\mathbb{R})\right)$ are conjugate it follows that the latter is surjective. Moreover, since $\mathscr{D}^{\prime}(\mathbb{R})$ and $\mathscr{D}^{\prime}(Y)$ are topologically isomorphic (see [29]) we finally obtain surjectivity of $P(D)$ on $\mathscr{D}^{\prime}\left(X, \mathscr{D}^{\prime}(Y)\right)$ which proves part i) of b).

For a polynomial $P$ which acts along a subspace and is elliptic there, the same arguments used to prove b) i) yield part ii) of b).

Finally, let $P(x)=\alpha \prod_{j=1}^{l}\left(\left\langle N_{j}, x\right\rangle-c_{j}\right)$ such that $P(D)$ is surjective on $\mathscr{D}^{\prime}(X)$. By part b) of Theorem $18 \mathscr{D}_{P}^{\prime}(X)$ has $(P \Omega)$. Thus, applying [1, Proposition 8.3] and the Schwartz-Kernel Theorem once more gives b) iii).

\section{REFERENCES}

[1] J. Bonet, P. Domański, Parameter dependence of solutions of differential equations on spaces of distributions and the splitting of short exact sequences, J. Funct. Anal. 230:329-381, 2006.

[2] J. Bonet, P. Domański, The splitting of exact sequences of PLS-spaces and smooth dependence of solutionsof linear partial differential equations. Adv. Math. 217(2):561-585, 2008.

[3] P. Domański, Classical PLS-spaces: spaces of distributions, real analytic functions and their relatives. In Orlicz centenary volume, 51-70 Banach Center Publ. 64. Polish Acad. Sci., Warsaw, 2004.

[4] P. Domański, Real analytic parameter dependence of solutions of differential equations. Rev. Mat. Iberoam. 26(1):175-238, 2010.

[5] L. Frerick, T. Kalmes, Some results on surjectivity of augmented semi-elliptic differential operators, Math. Ann. 347:81-94, 2010.

[6] A. Grothendieck, Sur les espaces de solution d'une classe générale d'equations aux dérivées partielles, J. d'analyse math. 2:243-280, 1952-53.

[7] A. Grothendieck, Produits tensoriels topologiques et espaces nucléaires, Mem. Amer. Math. Soc. 16, 1955.

[8] L. Hörmander, On the range of convolution operators, Ann. of Math. 76 (1962), 148-170

[9] L. Hörmander, On the existence and the regularity of solutions of linear pseudo-differential equations, Ens. Math. 17:99-163, 1971.

[10] L. Hörmander, The Analysis of Linear Partial Differential Operators I and II, Springer, 1983.

[11] T. Kalmes, Every P-convex subset of $\mathbb{R}^{2}$ is already strongly P-convex. Math. Z. 269(3-4):721$731,2011$.

[12] T. Kalmes, Some results on surjectivity of augmented differential operators, J. Math. Anal. Appl. 386:125-134, 2012.

[13] T. Kalmes, The augmented operator of a surjective partial differential operator with constant coefficients need not be surjective, Bull. London Math. Soc. 44:610-614, 2012.

[14] B. Malgrange, Existence et approximation des solutions des équations aux dérivées partielles et des équations de convolution, Ann. Inst. Fourier Grenoble, 6 (1955-1956), 271-355

[15] R. Meise, B.A. Taylor, and D. Vogt, Characterization of the linear partial differential operators with constant coefficients that admit a continuous linear right inverse, Ann. Inst. Fourier (Grenoble), 40(3):619-655, 1990.

[16] R. Meise, B.A. Taylor, and D. Vogt, Continuous linear right inverses for partial differential operators of order 2 and fundamental solutions in half spaces. Manuscripta Math. 90(4):449464, 1996

[17] R. Meise, D. Vogt. Introduction to Functional Analysis, Clarendon Press, Oxford, 2004.

[18] S. Nakane, P-Convexity with respect to differential operators which act on linear subspaces, Proc. Japan Acad. 55 Ser. A: 343-347, 1979.

[19] J. Persson, The wave operator and P-convexity, Boll. Un. Mat. Ital. (5) 18-B: 591-604, 1981.

[20] J. Persson, The geometry of P-convex sets, Boll. Un. Mat. Ital. (7) 7-B: 549-573, 1993.

[21] K. Tintarev, On the geometry of P-convex sets for operators of real principle type, Israel J. Math. 64:195-206, 1988.

[22] K. Tintarev, Characterization of P-convexity for supports in terms of tangent curves, J. Math. Anal. Appl. 164:590-596, 1992.

[23] F. Trèves, Linear Partial Differential Operators with Constant Coefficients, Mathematics and its Applications. 6. New York etc.: Gordon and Breach Science Publishers. X, 1966.

[24] F. Trèves, Locally Convex Spaces and Linear Partial Differential Equations, Springer, 1967.

[25] F. Trèves, Topological Vector Spaces, Distributions, and Kernels, Academic Press, 1967.

[26] D. Vogt, Subspaces and quotients of (s). In Functional Analysis: Survey and Recent Results (K.D. Bierstedt and B. Fuchssteiner, ed.), North-Holland Math. Stud. 27, 167-187, 1977. 
[27] D. Vogt, M.J. Wagner, Charakterisierung der Quotientenräume von s und eine Vermutung von Martineau, Studia Math. 68:225-240, 1980.

[28] D. Vogt, On the Solvability of $P(D) f=g$ for vector valued functions, RIMS Kokyoroku 508:168-181, 1983.

[29] D. Vogt, Sequence space representations of spaces of test functions and distributions. In Functional analysis, holomorphy, and approximation theory (Rio de Janeiro, 1979), volume 83 of Lecture Notes in Pure and Appl. Math., pages 405-443. Dekker, New York, 1983.

[30] D. Vogt, Invariants and spaces of zero solutions of linear partial differential operators. Arch. Math. 87:163-171, 2006.

[31] G. Wiechert, Dualitäts- und Strukturtheorie der Kerne von linearen Differentialoperatoren, Dissertation Wuppertal, 1982.

[32] E.C. Zachmanoglou, An application of Holmgren's theorem and convexity with respect to differential operators with flat characteristic cones, Trans. Amer. Math. Soc. 140:109-115, 1969.

Technische Universität Chemnitz, Fakultät Für Mathematik, 09107 ChemNITZ, GERMANy

E-mail address: thomas.kalmes@mathematik.tu-chemnitz.de 